\title{
NOTE ON A PAPER OF TSUZUKU
}

\author{
by H. K. FARAHAT \\ (Received 6 November, 1963)
}

In [2], Tosiro Tsuzuku gave a proof of the following:

THEOREM. Let $G$ be a doubly transitive permutation group of degree $n$, let $K$ be any commutative ring with unit element and let $\rho$ be the natural representation of $G$ by $n \times n$ permutation matrices with elements 0,1 in $K$. Then $\rho$ is decomposable as a matrix representation over $K$ if and only if $n$ is an invertible element of $K$.

For $G$ the symmetric group this result follows from Theorems (2.1) and (4.12) of [1]. The proof given by Tsuzuku is unsatisfactory, although it is perfectly valid when $K$ is a field. The purpose of this note is to give a correct proof of the general case.

Let $M$ be the representation module realizing the representation $\rho$, that is, $M$ is the free left $K$-module generated by the permuted letters $e_{1}, \ldots, e_{n}$. Suppose that $n$ is invertible in $K$. Then for arbitrary elements $k_{i}$ of $K$ we have

$$
\sum_{i=1}^{n} k_{i} e_{i}=n^{-1} k \cdot e+\sum_{i=2}^{n}\left(k_{i}-n^{-1} k\right)\left(e_{i}-e_{1}\right)
$$

where $k=k_{1}+\ldots+k_{n}, e=e_{1}+\ldots+e_{n}$. This shows that $M$ is the direct sum of the representation submodules $K e$ and $\sum_{i=2}^{n} K\left(e_{i}-e_{1}\right)$.

Conversely, suppose that $M$ is the direct sum of two representation submodules: $M=M^{\prime}+M^{\prime \prime}$. By definition of representation modules, $M^{\prime}$ and $M^{\prime \prime}$ are $K$-free (this point was missed by Tsuzuku), and every $m \in M$ has a unique decomposition in the form $m=m^{\prime}+m^{\prime \prime}$, where $m^{\prime} \in M^{\prime}, m^{\prime \prime} \in M^{\prime \prime}$. Clearly $(g m)^{\prime}=g m^{\prime},(g m)^{\prime \prime}=g m^{\prime \prime}$ for $g \in G, m \in M$. Let $e_{i}^{\prime}=\sum_{j=1}^{n} \kappa\left(e_{i}, e_{j}\right) e_{j}$, where $\kappa\left(e_{i}, e_{j}\right) \in K$. If now $g$ is any permutation belonging to $G$, then

$$
\left(g e_{i}\right)^{\prime}=g e_{i}^{\prime}=\sum_{j=1}^{n} \kappa\left(e_{i}, e_{j}\right) g e_{j} .
$$

This shows that, for all $g \in G$,

$$
\kappa\left(e_{i}, e_{j}\right)=\kappa\left(g e_{i}, g e_{j}\right) \quad(i, j=1, \ldots, n) .
$$

Put $\kappa\left(e_{1}, e_{1}\right)=\lambda, \kappa\left(e_{1}, e_{2}\right)=\mu$. Since $G$ is doubly transitive, we have $\kappa\left(e_{i}, e_{i}\right)=\lambda$ (for all $i$ ) and $\kappa\left(e_{i}, e_{j}\right)=\mu$ for $i \neq j$. Hence, again writing $e=e_{1}+\ldots+e_{n}$, we get

$$
e_{i}^{\prime}=\mu e+\sigma e_{i}, \text { where } \sigma=\lambda-\mu \text {. }
$$

Hence $e^{\prime}=(n \mu+\sigma) e$ and therefore

$$
\left(e_{i}^{\prime}\right)^{\prime}=\mu(n \mu+2 \sigma) e+\sigma^{2} e_{i}
$$

But $\left(e_{i}^{\prime}\right)^{\prime}=e_{i}^{\prime} ;$ consequently

$$
\sigma^{2}=\sigma \text { and } \mu \chi=0 \text {, where } \chi=n \mu+2 \sigma-1 .
$$


From this and the fact that $\left(m^{\prime}\right)^{\prime \prime}=0$, we find that

$$
0=\left(\chi e_{i}^{\prime}\right)^{\prime \prime}=\left(\chi \sigma e_{i}\right)^{\prime \prime}=\chi \sigma \cdot e_{i}^{\prime \prime} \quad(i=1, \ldots, n) .
$$

This implies that $\chi \sigma M^{\prime \prime}=0$ and, as $M^{\prime \prime}$ is $K$-free, we deduce that $\chi \sigma=0$, and consequently $\chi e_{i}^{\prime}=0(i=1, \ldots, n)$. Again this shows that $\chi M^{\prime}=0$; whence $\chi=0$. We now have

$$
(n \mu)^{2}=(1-2 \sigma)^{2}=1-4 \sigma+4 \sigma^{2}=1,
$$

which proves that $n$ is an invertible element of $K$. This completes the proof.

\section{REFERENCES}

1. H. K. Farahat, On the natural representation of the symmetric groups, Proc. Glasgow Math. Assoc. 5 (1962), 121-136.

2. T. Tsuzuku, On decompositions of the permutation representation of a permutation group, Nagoya Math. J. 22 (1963), 79-82.

\section{THE UNIVERSITY}

SHEFFIELD 